\title{
Inhalt
}

Literaturverzeichnis $[1$ bis 21$] \ldots \ldots \ldots \ldots \ldots \ldots \ldots \ldots$

\section{Grundlagen der Festigkeitslehre}

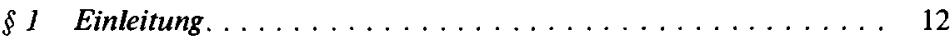

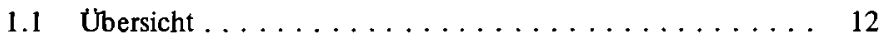

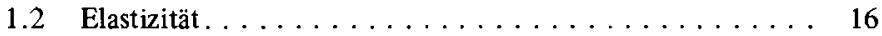

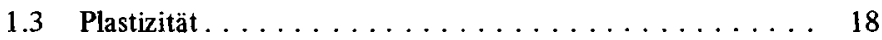

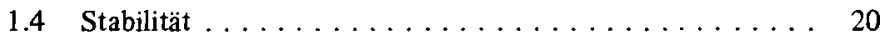

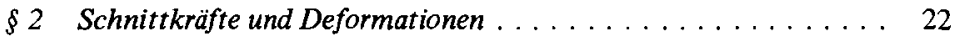

2.1 Statik und Kinematik des starren Körpers. . . . . . . 22

2.2 Arbeitsgleichung und Schnittkräfte . . . . . . . . . 24

2.3 Formänderungsenergie und Biegelinie $\ldots \ldots \ldots \ldots \ldots 26$

2.4 Differentielles Gleichgewicht dehnbarer Körper . . . . . . 35

2.5 Differentialgleichung der Balkenbiegung als Anfangswertproblem . . . . . . . . . . . . . . . 38

2.6 Differentialgleichung der Balkenbiegung als Randwertproblem . . . . . . . . . . . . . . . 44

2.7 Numerisches Gleichgewicht $\ldots \ldots \ldots \ldots \ldots \ldots \ldots \ldots . \ldots \ldots$

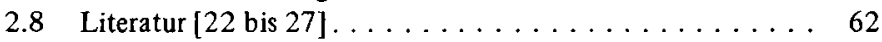

$\S 3$ Spannung und Verzerrung $\ldots \ldots \ldots \ldots \ldots \ldots \ldots \ldots \ldots$

3.1 Spannungszustand $\ldots \ldots \ldots \ldots \ldots \ldots \ldots \ldots \ldots$

3.2 Verzerrungszustand $\ldots \ldots \ldots \ldots \ldots \ldots \ldots \ldots$

\$ 4 Elastizitätsgesetze und Fließbedingungen $\ldots \ldots \ldots \ldots \ldots \ldots$

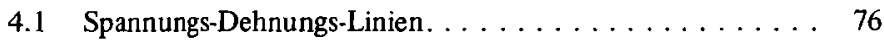

4.2 Elastische und plastische Hysteresis . . . . . . . . . 86

4.3 Hooke'sches Gesetz und elastische Grundgleichungen . . . . 87

4.4 Fließbedingungen und Verfestigungshypothesen....... 91

4.5 Schrifttum $[28$ bis 47$] \ldots \ldots \ldots \ldots \ldots \ldots \ldots$

5 Energie- und Extremalprinzipien . . . . . . . . . . . 99

5.1 Prinzip der virtuellen Arbeit . . . . . . . . . . . . . 99

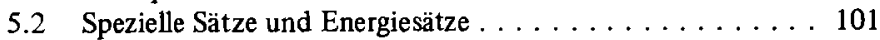

5.3 Sätze zur Berechnung der Traglast . . . . . . . . . . 103 
5.4 Sätze für stabiles Werkstoff verhalten und für das Einspielen (shake-down) . . . . . . . . . . . . . . . 105

5.5 Schrifttum $[48$ bis 67$] \ldots \ldots \ldots \ldots \ldots \ldots \ldots$

\section{Zug und Druck}

$\$ 6$ Seile, Ringe und Behälter. . . . . . . . . . . . . . . 107

6.1 Seile . . . . . . . . . . . . . . . . . . . . 107

6.2 Ringe und Behälter. . . . . . . . . . . . . . 116

6.3 Silodruck . . . . . . . . . . . . . . . . . . . . . . 119

6.4 Schrifttum $[68$ bis 80$] \ldots \ldots \ldots \ldots \ldots \ldots \ldots \ldots$

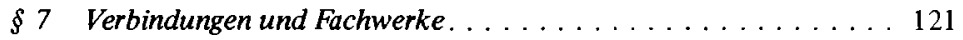

7.1 Verbindungstechnik ................. 121

7.2 Elastische und plastische Fachwerke . . . . . . . . 125

7.3 Schrifttum $[81$ bis 93$] \ldots \ldots \ldots \ldots \ldots \ldots \ldots$

\section{Balkenbiegung}

$\S 8$ Querschnittsfestigkeit . . . . . . . . . . . . . . 133

8.1 Querschnittswerte . . . . . . . . . . . . . 133

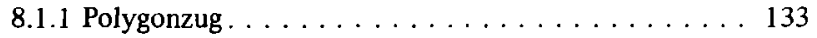

8.1.2 Diskretisierung . . . . . . . . . . . . . . 138

8.2 Elastische Biegung ohne Normalkraft . . . . . . . . . . . 139

8.2.1 Allgemeiner Querschnitt . . . . . . . . . . . . . . 139

8.2 .2 Verbundquerschnitt . . . . . . . . . . . . . 142

8.2.3 Schubspannungen und Schubmittelpunkt . . . . . . . 143

8.3 Elastische Biegung mit Normalkraft . . . . . . . . . . 148

8.3.1 Kern des Querschnittes . . . . . . . . . . . . . . 149

8.3.2 Druckspannungen bei versagender Zugzone . . . . . 152

8.3.3 Kerbspannungen . . . . . . . . . . . . . . 154

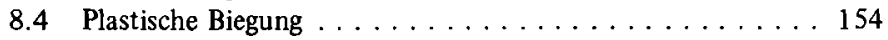

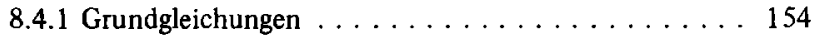

8.4.2 Symmetrische Biegung ohne Normalkraft . . . . . . 157

8.4.3 Biegung mit Normalkraft . . . . . . . . . . . . . 164

8.5 Schrifttum $[94$ bis 102$] \ldots \ldots \ldots \ldots \ldots \ldots \ldots$

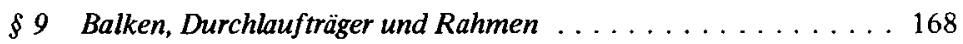

9.1 Biegelinie statisch bestimmter Systeme. . . . . . . . . 168

9.1.1 Elastische und plastische Biegung . . . . . . . . . 169

9.1.2 Schubspannungen bei der plastischen Biegung . . . . 172 
9.2 Statisch unbestimmte Balken und Durchlaufträger . . . . . . 174

9.2.1 Eingespannte Träger . . . . . . . . . . . . . 175

9.2.2 Ein Paradoxon der Traglast theorie. . . . . . . . . 180

9.2.3 Elastische Berechnung und Dreimomentengleichung . . 182

9.2.4 Plastische Berechnung des Durchlaufträgers. . . . . . 184

9.3 Rahmen. . . . . . . . . . . . . . . . . . 188

9.3.1 Elastische Berechnung. . . . . . . . . . . . 188

9.3.2 Plastische Berechnung. . . . . . . . . . . . 190

9.4 Schrifttum $[103$ bis 111$] \ldots \ldots \ldots \ldots \ldots \ldots . \ldots \ldots$

$\S 10$ Hängebrücken und Bogenbrücken . . . . . . . . . . . . . . 193

10.1 Hängebrücken nach der Theorie 2. Ordnung . . . . . . . 193

10.2 Zweigelenkbogenbrücken nach der Theorie 2. Ordnung . . . 199

10.3 Zahlenbeispiel . . . . . . . . . . . . . . 201

10.4 Schrifttum $[112$ bis 123$] \ldots \ldots \ldots \ldots \ldots \ldots$

$\$ 11$ Elastische Bettung . . . . . . . . . . . . . . 206

11.1 Steifezahlverfahren . . . . . . . . . . . . . 207

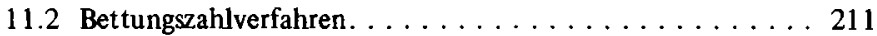

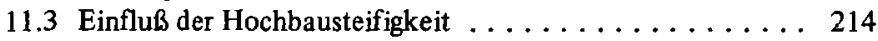

11.4 Schrifttum $[124$ bis 158$] \ldots \ldots \ldots \ldots \ldots \ldots . \ldots \ldots$

\section{Knickfestigkeit}

$\$ 12$ Knickung . . . . . . . . . . . . . . . . . . 217

12.1 Knickung im elastischen Bereich . . . . . . . . 218

12.2 Knickung im überkritischen Bereich. . . . . . . . . . 223

12.3 Knickung im plastischen Bereich . . . . . . . . . . . 224

12.4 Kippen. . . . . . . . . . . . . . . . . . . . . 226

12.5 Schrifttum $[159$ bis 199$] \ldots \ldots \ldots \ldots \ldots \ldots . \ldots 231$

$\S 13$ Knickbiegung . . . . . . . . . . . . . . . . 233

13.1 Balkendruckbiegung . . . . . . . . . . . . . 234

13.2 Stockwerkrahmen . . . . . . . . . . . . . . . 241

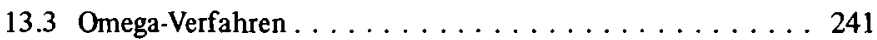

13.4 Schrifttum $[200$ bis 210$] \ldots \ldots \ldots \ldots \ldots \ldots . \ldots \ldots 243$

Sachverzeichnis . . . . . . . . . . . . . . . . . . 245

Namensverzeichnis . . . . . . . . . . . . . . . . . . . 247 
Inhalt des zweiten Bandes

Elastizität, Plastizität und Stabilität der Flächentragwerke

V Neuere Berechnungsmethoden

$\S 14$ Operatorenrechnung

$\S 15$ Matrizen- und Tensoralgebra

$\S 16$ Elementenmethode

VI Torsion

$\S 17$ Elastische Torsion

$\S 18$ Plastische Torsion

VII Membranen, Platten, Scheiben

$\S 19$ Membranen

$\S 20$ Platten

$\S 21$ Scheiben

\section{Schalen}

$\S 22$ Translations- und Rotationsschalen

$\S 23$ Einfach gekrümmte Schalen und Faltwerke

\section{Stabilitätsprobleme}

§ 24 Plattenbeulung

$\S 25$ Schalenbeulung 